\title{
Pre-vaccination testing could expand coverage of two-dose
}

\section{COVID vaccines [version 1; peer review: 1 approved, 1}

\section{approved with reservations]}

\author{
Carl A. B. Pearson (D1,2, Samuel Clifford (D1), Juliet R. C. Pulliam (D2, \\ Rosalind M. Eggo (D)1 \\ ${ }^{1}$ Infectious Disease Epidemiology \& Centre for Mathematical Modelling of Infectious Disease, London School of Hygiene \& Tropical \\ Medicine, Keppel Street, London, WC1E 7HT, UK \\ 2DSI-NRF Centre of Excellence in Epidemiological Modelling and Analysis, Stellenbosch University, 19 Jonkershoek Road, \\ Stellenbosch, 7600, South Africa
}

V1 First published: 11 May 2021, 6:105

https://doi.org/10.12688/wellcomeopenres.16835.1

Latest published: 11 May 2021, 6:105

https://doi.org/10.12688/wellcomeopenres.16835.1

\begin{abstract}
Recent evidence indicates that a single dose of mRNA-based vaccines produce similar immune responses in people with evidence of past infection compared with two doses in immunologically naive individuals. For COVID-19 vaccines with two dose regimens, point-ofcare antibody testing for prior infection when administering the first dose could enable expanded vaccine access in a cost-effective manner. Generally, antibody tests with sensitivity and specificity well below that typically accepted for product licensure would still enable expanded vaccine coverage, though to be cost-beneficial total test cost (i.e. procurement and administration) needs to be less than roughly a third of total vaccine dose cost. For highly sensitive (90\%) and specific ( $99 \%$ ) tests, coverage could be expanded by more than $33 \%$. Tests with the appropriate performance characteristics are plausible, though likely need setting specific tailoring.
\end{abstract}

Keywords

COVID-19, SARS-CoV-2, Vaccination, Point-of-Care Antibody Testing, Rapid Serological Testing

\section{Open Peer Review}

Approval Status

$?$

1

2

version 1

11 May 2021

$?$

1. Thomas Crellen $(D)$, University of Oxford, Oxford, UK

2. Ana Bento ID, Indiana University School of Public Health-Bloomington, Bloomington, USA

Any reports and responses or comments on the article can be found at the end of the article. 
Corresponding author: Carl A. B. Pearson (carl.pearson@lshtm.ac.uk)

Author roles: Pearson CAB: Conceptualization, Formal Analysis, Investigation, Methodology, Software, Visualization, Writing - Original Draft Preparation, Writing - Review \& Editing; Clifford S: Conceptualization, Validation, Visualization, Writing - Original Draft Preparation, Writing - Review \& Editing; Pulliam JRC: Conceptualization, Formal Analysis, Methodology, Supervision, Validation, Visualization, Writing - Original Draft Preparation, Writing - Review \& Editing; Eggo RM: Methodology, Project Administration, Resources, Supervision, Visualization, Writing - Original Draft Preparation, Writing - Review \& Editing

Competing interests: No competing interests were disclosed.

Grant information: This research was partly funded by the Bill and Melinda Gates Foundation (NTD Modelling Consortium OPP1184344: CABP), FCDO/Wellcome Trust (221303: CABP; 208812: SC), HDR UK (MR/S003975/1: RME), UK MRC (MCI_PCI_19065: RME). JRCP is supported by the South African Department of Science and Innovation and the National Research Foundation (NRF). Any opinion, finding, and conclusion or recommendation expressed in this material is that of the authors and the NRF does not accept any liability in this regard.

The funders had no role in study design, data collection and analysis, decision to publish, or preparation of the manuscript.

Copyright: (c) 2021 Pearson CAB et al. This is an open access article distributed under the terms of the Creative Commons Attribution License, which permits unrestricted use, distribution, and reproduction in any medium, provided the original work is properly cited.

How to cite this article: Pearson CAB, Clifford S, Pulliam JRC and Eggo RM. Pre-vaccination testing could expand coverage of twodose COVID vaccines [version 1; peer review: 1 approved, 1 approved with reservations] Wellcome Open Research 2021, 6:105 https://doi.org/10.12688/wellcomeopenres.16835.1

First published: 11 May 2021, 6:105 https://doi.org/10.12688/wellcomeopenres.16835.1 


\section{Introduction}

Many countries have experienced high SARS-CoV-2 infection attack rates and currently have limited vaccine supplies. For example, countries participating in the CO-VAX facility expect to receive vaccine coverage for only $20 \%$ of their populations $^{1}$, with any additional coverage depending on availability and cost. However, recent evidence indicates that a single dose of the mRNA-based vaccines (e.g. the Pfizer or Moderna products) produce immune responses in people with evidence of past infection (i.e. seropositive) that are at least comparable to the response elicited by two doses in immunologically naive individuals ${ }^{2-6}$.

If effects observed in these studies are validated, the use of a point-of-care antibody test when administering the first dose could allow for more efficient use of available vaccine doses. This benefit depends on test performance, cost, and the seroprevalence in the population being vaccinated. Intuitively, the advantage will increase with greater seroprevalence because there is more opportunity to reallocate the second dose. To be a better option than buying more doses, administering a test should cost less than a single vaccine dose, but how much less will depend on test specificity (to avoid giving too few doses) and sensitivity (to benefit from reallocating doses), as well as seroprevalence. Several rapid, point-of-care antibody tests $^{7-10}$ are available that may ultimately be able meet these requirements; however, further testing and development may be needed to validate and tailor tests for use in different contexts $^{11-13}$. Data to evaluate vaccine effectiveness for a single dose conditional on antibody testing could be collected in countries pursuing a delayed second dose scheme, like the United Kingdom.

\section{Methods}

Calculations performed in $\mathrm{R}(4.0 .3)^{14}$, with visualizations prepared using data.table ${ }^{15}$, ggplot $2^{16}$, and patchwork ${ }^{17}$.

\section{Coverage with testing}

To analyze the direct benefit of this dose-sparing approach, we conservatively assume seronegative individuals are fully vaccinated (i.e. protected at the efficacy of the vaccine) only after two doses, and seropositive individuals after one dose. With a test, most seropositive individuals get one dose instead of two, freeing up doses to protect more people. Given a fixed supply, the number of additional people that could be protected depends on both test performance and the seroprevalence in the population of interest.

To evaluate this relationship, we model the population as having a homogeneous distribution of potentially test-detectable prior SARS-CoV-2 infection, characterized by a population-level seroprevalance, $\rho_{+}=1-\rho_{-}$. We model a test that is characterized by sensitivity (the true-positive rate, the complement of the false negative rate TPR $=1-$ FNR), and specificity (the true-negative rate, the complement of the false positive rate $\mathrm{TNR}=1-\mathrm{FPR})$. For practical applications, these values will be entangled, since we know that e.g. SARS-CoV-2 antibody responses decline with time ${ }^{18}$.
If we assume, pessimistically, that a single dose is not protective when given to seronegative individuals, then in our model the people protected per dose (PPD) is:

$$
\mathrm{PPD}=0.5 *\left(\mathrm{TNR} \rho_{-}+\mathrm{FNR} \rho_{+}\right)+1 * \mathrm{TRP} \rho_{+}+0 * \mathrm{FRP} \rho_{-}
$$

which we can re-arrange in terms of complementary parameters:

$$
\begin{aligned}
2 \mathrm{PPD} & =\left(\operatorname{TNR}\left(1-\rho_{+}\right)+(1-\mathrm{TRP}) \rho_{+}\right)+2 \mathrm{TRP} \rho_{+} \\
& =\mathrm{TNR}-\mathrm{TNR} \rho_{+}+\rho_{+}-\mathrm{TRP} \rho_{+}+2 \mathrm{TRP} \rho_{+} \\
& =\mathrm{TNR}-\mathrm{TNR} \rho_{+}+\rho_{+}+\mathrm{TRP} \rho_{+} \\
& =\mathrm{TNR}+\rho_{+}(1+\mathrm{TRP}-\mathrm{TNR})
\end{aligned}
$$

Recall that without testing, two doses are required for vaccine immunity, i.e. $\mathrm{PPD}=0.5$. This corresponds to the situation where $\mathrm{TNR}=1$ (because the "test" is simply that everyone gets two doses) and TPR $=0$ (because no one gets a single dose). Relative to this baseline, the relative change in PPD due to introducing testing is:

$$
\begin{aligned}
\Delta_{\mathrm{PPD}}= & \frac{2 \mathrm{PPD}\left(\mathrm{TNR}, \mathrm{TRP}, \rho_{+}\right)}{2 \operatorname{PPD}(1,0, \sim)}-1 \\
& =\mathrm{TNR}+\rho_{+}(1+\mathrm{TPR}-\mathrm{TNR})-1
\end{aligned}
$$

Assuming a single dose is non-protective in seronegative individuals helps ensure that the model represents the minimal benefit, and thus decisions continue to be beneficial even after accommodating real-world factors that are impractical to model. This limiting assumption implies that introducing testing can reduce the number of people effectively vaccinated. As practical matter, this is only a problem with extreme combinations of sero-prevalence and test performance, which are not generally relevant for the settings where this scheme is worth considering. The constraining relation is $\Delta_{\mathrm{PPD}}>0$ or $\mathrm{TNR}+\rho_{+}(1+\mathrm{TPR}-\mathrm{TNR})>1$. For example, in a population with $20 \%$ seroprevalence, using a test with low specificity, $50 \%$, would be detrimental even with a perfectly sensitive test.

\section{Costs}

Though testing will increase the overall cost of the vaccine program (relative to a fixed number of available vaccine doses), decision-makers should consider the cost per fully vaccinated person. One way to make that decision is to estimate which is the less expensive way to expand coverage: expanding the supply by buying more vaccine doses or more efficiently using the existing supply with testing. We model the total cost per vaccine dose of $V$ (i.e. production, logistics, and administration) and total cost per test of $T$. On the margin, imagine purchasing one more protected person: that can be accomplished for $2 V$ (i.e. two doses to get a fully vaccinated person without testing) or for some expected number of tests, $n T$, to allow re-allocation of unnecessary second doses from 
seropositive recipients. Restricting our consideration to situations with positive $\Delta_{\text {PPD }}$, we can think about the relationship between $n$ and $\Delta_{\mathrm{PPD}}$.

If testing increases the PPD by e.g. 10\%, then using 20 tests (on average) would add another fully immunized individual. Even in a situation where the test is perfect and everyone is seropositive (i.e. testing increases PPD by 100\%), two tests are still required: one to free up the second dose from an individual and one to confirm that the next recipient in line only needs one. We can generalize this relationship to $n=\frac{2}{\Delta_{\mathrm{PPD}}}$. Thus,
using tests is preferred when

$$
\frac{2}{\Delta_{\mathrm{PPD}}} \leq 2 \mathrm{~V} \rightarrow \frac{T}{V} \leq \Delta_{\mathrm{PPD}}
$$

\section{Results}

\section{Expanded coverage}

Using Equation 2, we find that using a pre-vaccination antibody testing program could substantially expand vaccine coverage in populations with relatively high seroprevalence. Rearranging Equation 2 and considering a high specificity $(99 \%$ or TNR $=0.99)$ test, only moderate seroprevalence and sensitivity are required to observe gains:

$$
\rho_{+}(0.01+\mathrm{TPR})>0.01
$$

With this high specificity and a $40 \%$ seroprevalence $\left(\rho_{+}=0.4\right)$ in the population of interest, only TPR $>0.015$ (or sensitivity of $1.5 \%$ ) is necessary to expand coverage, though of course such low performance does not expand coverage much. However, a test with sensitivity more typical of licensed medical assays (90\%) would expand coverage by $35 \%$ (e.g. increasing a COVAX-like coverage of $20 \%$ to $27 \%$ ). Given that the test is not needed to determine treatment, but rather more efficiently allocate doses, lower sensitivity (e.g. 70\%) might be substantially less expensive, and even that lower performance would expand coverage by $27 \%$ (e.g. increasing a COVAX-like coverage of $20 \%$ to $25 \%$ ). In each of the three dimensions, coverage gains increase linearly: higher seroprevalence means more opportunity to reallocate doses, higher sensitivity means taking better advantage of that opportunity, and higher specificity decreases the (assumed) loss of protection. Figure $1 \mathrm{~A}$ shows this trend for seroprevalence (moving left to right on plot) and sensitivity (shifting from lower to higher trend lines).

\section{Paying for testing versus more doses}

Similar to expanding coverage, using Equation 3, we find that regions with relatively high seroprevalence could more effectively expand coverage with antibody testing than additional doses with a relatively high threshold for test cost. Per Equation 3, the cost threshold is identical to the coverage expansion, and as such also increases linearly with seroprevalence and test performance. Figure $1 \mathrm{~B}$ shows this trend for
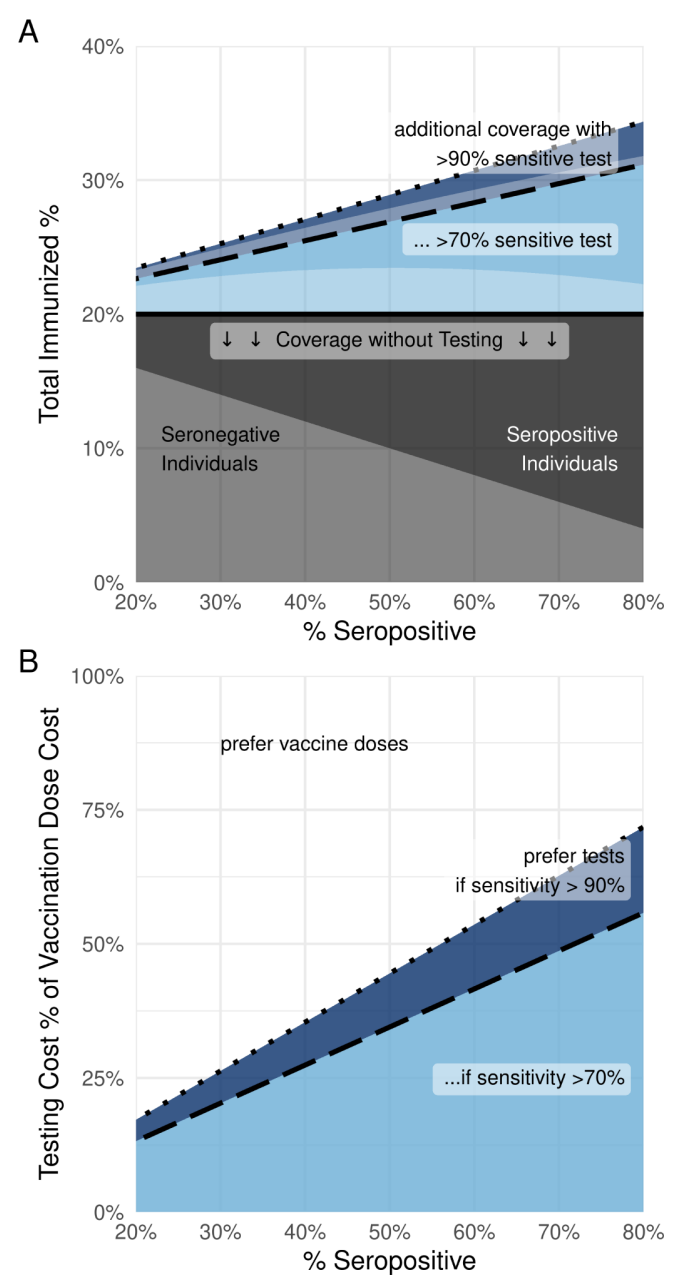

Figure 1. Example benefits of testing. A) Assuming roughly $20 \%$ of the population (light grey region) could be vaccinated without any testing: with a highly specific test (99\%), testing can expand the percentage covered by increasing amounts depending on seroprevalence and test sensitivity (light blue: 70\% sensitive, dark blue: $90 \%$ sensitivity). While many individuals still go unvaccinated (white background), further incremental vaccine supply would result in similarly extended coverage under pre-vaccination testing approach. B) Testing can also expand the vaccinated fraction for lower costs, again depending on the vaccine-eligible population's seroprevalence and the test's performance. For a highly specific test, and relatively high seroprevalence, e.g. $40 \%$, a test that is less than $27 \%$ (if $>70 \%$ sensitive) to $35 \%$ (if $>90 \%$ sensitive) the cost of a vaccine dose is a cheaper way to expand coverage than buying more doses of vaccine. The cost advantage of testing grows with increasing seroprevalence and test performance.

seroprevalence (moving left to right on plot) and sensitivity (shifting from lower to higher trend lines).

Recalling the high seroprevalence (40\%), high specificity (99\%), and high sensitivity (90\%) scenario, the cost threshold would be $35 \%$ of the cost of vaccination. Considering a per dose cost of roughly 15 USD (a rough direct price for the 
mRNA vaccines ${ }^{19}$, but ignoring the additional administration and logistics costs), a test that cost around 5 USD or less would be a better investment to expand coverage. Even at a lower sensitivity $(70 \%)$, the cost threshold remains plausible at 4 USD or less per test.

\section{Discussion and conclusions}

Where a pre-vaccination antibody testing programme is implemented, vaccine administration could be adapted to include a rapid, point-of-care antibody test before the first dose. The test result could be delivered at the end of the normal monitoring period for severe acute reactions in recipients, allowing them to be advised whether they need a follow-up dose based on their test result. For tests with imperfect specificity, some individuals with no past infection history would only receive a single dose; for our analysis, we conservatively assume these people get no protection, though studies indicate at least short-term protection ${ }^{2}$. Other studies have suggested that past exposure alone is sufficiently protective, and that testing could be used to administer vaccines only to seronegative individuals ${ }^{20}$. While theoretically plausible, such an approach seems practically and politically untenable, could exacerbate existing inequity in COVID-19 health burdens, and does not account for potential effects like waning immunity or immune escape variants where a single dose may boost protection $^{3,6}$.

We have framed this analysis in terms of a population of interest. While that population could be an entire country, it could likewise be a sub-national region and/or a particular age or employment group. The pre-vaccination testing approach is most beneficial for populations with high prior burden where there is still high risk of infection, but improperly communicated could lead to the perception of these groups receiving inferior treatment and further exacerbating health inequities. While increased vaccine availability or more globally equitable distribution would reduce the need for dose-sparing methods, pragmatic solutions to expand coverage like testing may enable more efficient use in the current vaccine context. Indeed, approaches like this could be adopted in higher-income settings, where testing may require less calibration and other infrastructure advantages could offset the costs, thus freeing up vaccine supply globally.

In this analysis, we have tried to offer a conservative estimate of the potential benefits of using testing. We have focused on the directly protected individuals and the direct costs, but expanding vaccination could also increase indirect protection by reducing transmission broadly ${ }^{21}$. Likewise, expanded vaccination could reduce the incidence of severe disease and associated costs and consumption of health service resources. We expect that a more sophisticated model incorporating these kinds of effects will predict higher testing benefits, though there are additional nuances that would dampen returns (e.g. accounting for prioritization of older populations who have high disease risk but likely lower past exposure rates). Given that our approach indicates substantial potential benefits despite being conservative, we recommend further development of rapid, point-of-care antibody testing and vaccine effectiveness studies to verify protection of seropositive individuals after single doses.

\section{Data availability}

All data underlying the results are available as part of the article and no additional source data are required.

\section{Code availability}

Source code available from https://github.com/cmmid/covidTestVac

Archived source code at time of publication: https://doi. org/10.5281/zenodo. $4725815^{22}$.

License: MIT
1. Gavi COVAX AMC Reference Source

2. Krammer F, Srivastava K, Alshammary H, et al.: Antibody Responses in Seropositive Persons after a Single Dose of SARS-CoV-2 mRNA Vaccine. N EnglJ Med. 2021; 384(14): 1372-1374.

PubMed Abstract | Publisher Full Text | Free Full Text

3. Stamatatos $\mathrm{L}$, Czartoski J, Wan $\mathrm{YH}$, et al:: mRNA vaccina tion boosts crossvariant neutralizing antibodies elicited by SARS-CoV-2 infection. Science. 2021; eabg9175.

PubMed Abstract | Publisher Full Text

4. Samanovic MI, Cornelius AR, Wilson JP, et al.: Poor antigen-specific responses to the second BNT162b2 mRNA vaccine dose in SARS-CoV-2-experienced individuals. medRxiv preprint. 2021. Publisher Full Text

5. Saadat S, Tehrani ZR, Logue J, et al.: Binding and Neutralization Antibody Titers After a Single Vaccine Dose in Health Care Workers Previously Infected With SARS-CoV-2. JAMA. 2021; 325(14): 1467-1469. PubMed Abstract | Publisher Full Text | Free Full Text

6. Lustig Y, Nemet I, Kliker L, et al.: Neutralizing Response against Variants after SARS-CoV-2 Infection and One Dose of BNT162b2. N EnglJ Med. 2021; NEJMc2104036.

PubMed Abstract | Publisher Full Text | Free Full Text

7. Baraniuk C: Covid-19 antibody tests: a briefing. BMJ. 2020; 369: m2284. PubMed Abstract | Publisher Full Text

8. Mathur G, Mathur S: Antibody Testing for COVID-19. Am J Clin Pathol. 2020; 154(1): 1-3.

PubMed Abstract | Publisher Full Text | Free Full Text

9. Shuren J, Stenzel T: The FDA's Experience with Covid-19 Antibody Tests. N EnglJ Med. 2021; 384(7): 592-594.

PubMed Abstract | Publisher Full Text

10. Prazuck $T$, Van JP, Sinturel F, et al.: Evaluation of the practicability of a fingerstick whole-blood SARS-Cov-2 self-test adapted for the general population. PLoS One. 2021; 16(1): e0245848.

PubMed Abstract | Publisher Full Text | Free Full Text

11. Ndaye AN, Hoxha A, Madinga J, et al.: Challenges in interpreting SARS-CoV-2 serological results in African countries. Lancet Glob Health. 2021; 9(5): e588-e589.

PubMed Abstract | Publisher Full Text | Free Full Text 
12. Tso FY, Lidenge SJ, Peña PB, et al.: High prevalence of pre-existing serological cross-reactivity against severe acute respiratory syndrome coronavirus-2 (SARS-CoV-2) in sub-Saharan Africa. Int J Infect Dis. 2021; 102: 577-583. PubMed Abstract | Publisher Full Text | Free Full Text

13. Emmerich P, Murawski C, Ehmen C, et al.: Limited specificity of commercially available SARS-CoV-2 IgG ELISAs in serum samples of African origin. Trop Med Int Health. 2021.

PubMed Abstract | Publisher Full Text | Free Full Text

14. R Core Team: R: A Language and Environment for Statistical Computing. R Foundation for Statistical Computing, Vienna, Austria, 2020. Reference Source

15. Dowle M, Srinivasan A: data.table: Extension of 'data.frame'. R package version 1.13.6. 2020. Reference Source

16. Wickham H: ggplot2: Elegant Graphics for Data Analysis. Springer-Verlag New York, R package version 3.3.3. 2016.

Reference Source

17. Pedersen TL: patchwork: The Composer of Plots. R package version 1.1.1.
2020.

Reference Source

18. Harris RJ, Whitaker HJ, Andrews NJ, et al:: Serological surveillance of sars-cov-2: Six-month trends and antibody response in a cohort of public health workers. J Infect. 2021; 82(5): 162-169.

PubMed Abstract | Publisher Full Text | Free Full Text

19. Dyer O: Covid-19: Countries are learning what others paid for vaccines. $B M$ J. 2021; 372: n281.

PubMed Abstract | Publisher Full Text

20. Bubar KM, Reinholt K, Kissler SM, et al: Model-informed COVID-19 vaccine prioritization strategies by age and serostatus. Science. 2021; 371(6532): prioritizatic

PubMed Abstract | Publisher Full Text | Free Full Text

21. Regev-Yochay G, Amit S, Bergwerk $\mathrm{M}$, et al.: Decreased Infectivity Following BNT162b2 Vaccination. SSRN Electronic Journal. 2021. Publisher Full Text

22. Pearson CAB: cmmid/covidtestvac: submission. 2021. http://www.doi.org/10.5281/zenodo.4725815 


\section{Open Peer Review}

\section{Current Peer Review Status:}

\section{Version 1}

Reviewer Report 02 July 2021

https://doi.org/10.21956/wellcomeopenres.18570.r43828

(C) 2021 Bento A. This is an open access peer review report distributed under the terms of the Creative Commons Attribution License, which permits unrestricted use, distribution, and reproduction in any medium, provided the original work is properly cited.

\section{Ana Bento}

Department of Epidemiology and Biostatistics, Indiana University School of Public HealthBloomington, Bloomington, IN, USA

I really enjoyed this study. In the current context, where vaccine availability is very different depending on the country, this can potentially have real applications to public health interventions and prioritization. It is very simple but elegant.

As a further step, I would introduce heterogeneity in susceptibility, test seeking and eventually introduction of VOCs.

Is the work clearly and accurately presented and does it cite the current literature? Yes

Is the study design appropriate and is the work technically sound?

Yes

Are sufficient details of methods and analysis provided to allow replication by others? Yes

If applicable, is the statistical analysis and its interpretation appropriate? Yes

Are all the source data underlying the results available to ensure full reproducibility? Yes

Are the conclusions drawn adequately supported by the results? Yes

Competing Interests: No competing interests were disclosed. 
Reviewer Expertise: mathematical models of infectious disease. Vaccine implementation design

I confirm that I have read this submission and believe that I have an appropriate level of expertise to confirm that it is of an acceptable scientific standard.

Reviewer Report 01 June 2021

https://doi.org/10.21956/wellcomeopenres.18570.r43825

(C) 2021 Crellen T. This is an open access peer review report distributed under the terms of the Creative Commons Attribution License, which permits unrestricted use, distribution, and reproduction in any medium, provided the original work is properly cited.

\section{Thomas Crellen}

Big Data Institute, University of Oxford, Oxford, UK

This short report provides a quantitative assessment of when it is cost effective to use a single vaccine dose plus an antibody test, instead of two vaccine doses, under the assumption that these provide comparable immune responses across the population. Overall the methods and analysis are appropriate for a short paper.

I have a few minor comments.

1. In terms of the "real world application" of this approach, the population will be heterogeneous in terms of past exposure and immune response following infection $+a$ single dose. A key heterogeneity will be age. Does past infection + a single vaccine dose provide a comparable antibody response to 2 doses in very old (e.g. $80+$ ) individuals?

2. Could a bit more thought on how population heterogeneity would affect the analysis / results be added to the introduction or discussion?

3. Another important consideration is whether the duration of immunity from 1 dose + past infection is comparable to that from 2 doses.

4. The results could be much better presented in terms of providing a "key results table". I find Figure 1 quite unclear and I suggest that this could be improved given that it is the only Figure.

5. For panel A, I don't find the comparison between blanket 2 dose vaccination and testing + vaccination very clear. Additionally, where does the assumed $20 \%$ coverage without testing come from? Why is the proportion of the population vaccinated considered only up to a maximum of $30 \%$ ? A seroprevalence of $>50 \%$ also seems implausible - are there any locations where COVID seroprevalence surveys are this high?

6. For Panel B, I would expect the "Testing cost \% of vaccinated dose cost" to decrease with higher seroprevalence, but the opposite is shown on the Figure. It's unclear to me how the coloured areas relate to test sensitivity. 
7. Overall I would encourage the presentation of results to be much clearer. E.g. a table or figure which shows for different antibody test sensitivities and seroprevalence how the cost effectiveness of testing +1 dose compares with blanket 2 dose vaccination.

Is the work clearly and accurately presented and does it cite the current literature? Partly

Is the study design appropriate and is the work technically sound? Partly

Are sufficient details of methods and analysis provided to allow replication by others? Yes

If applicable, is the statistical analysis and its interpretation appropriate? Yes

Are all the source data underlying the results available to ensure full reproducibility? Yes

Are the conclusions drawn adequately supported by the results? Yes

Competing Interests: No competing interests were disclosed.

Reviewer Expertise: Infectious disease epidemiology, pathogen genomics, statistical modelling. I confirm that I have read this submission and believe that I have an appropriate level of expertise to confirm that it is of an acceptable scientific standard, however I have significant reservations, as outlined above. 